\title{
Vibration and Noise Control and Emission of Marine Diesel Engine
}

\author{
Zhiqiang Zhao ${ }^{1, a^{*}}$ \\ ${ }^{1}$ Binzhou Vocational College, Binzhou, Shandong Province 256600 \\ a103937818@qq.com \\ * The corresponding author
}

\begin{abstract}
Keywords: Marine diesel engines; Vibration and noise; Control and emissions; Preventive measures
\end{abstract}

\begin{abstract}
As an important noise pollution, the main source of ship noise is the marine diesel engine. At present, with the conservation of the global economy, some achievements have been made in the control of exhaust emissions and heat recovery, so the community has begun to pay attention to pollution. Based on this, this paper will analyze the noise control and discharge of vibration of marine diesel engine through two concrete examples of noise failure, and give some preventive measures.
\end{abstract}

\section{Introduction}

Marine aircraft in the abnormal failure refers to the equipment during operation due to internal parts of the operation of the equipment caused by a certain sound. These sounds can come from diesel engine knocking cylinder, pull cylinder, supercharger surge, valve mechanism percussion, diesel engine attached to the structure. Marine diesel engine abnormal sound also means that there is a certain internal diesel engine failure, and this problem continues to intensify. In order to ensure the timely detection of noise problems, use the ear to listen and judge in the maintenance is the most direct and effective way.

\section{Introduction to Vibration and Noise of Marine Diesel Engine}

This part mainly analyzes the related contents of vibration and noise of marine diesel engine, and starts from the classification and principle of vibration noise.

Classification of Noises. There are many sources of the diesel engine's vibration noises, and the cause is very complex. These vibrations have an important impact on the life of the diesel engine's internal equipment and the overall performance of the diesel engine [1]. According to the work of diesel engines, diesel engine noise can be divided into the following categories: (1) mechanical noise. The main source of this noise is the gas pressure caused by the equipment in the course of the operation of the imbalance, due to vibration caused by internal impact and then form a noise; (2) combustion noise. (3) noise due to airflow. (3) noise generated by the combustion of the cylinder, piston, connecting rod and other parts of the gas generated during the combustion of the fuel in the diesel engine cylinder; When the diesel engine is in operation, the airflow in the exhaust duct will form noise by movement with the pipe.

Mechanism and Characteristics. In the study of ship diesel noise, aerodynamic noise and mechanical noise have undergone a certain degree of development, but the development of combustion noise began to attract people's attention. It is found that combustion noise is another important source of noise after mechanical noise in ship diesel engine noise research. The following is a brief introduction to the main mechanism of combustion noise: fuel combustion in the cylinder, the cylinder due to rapid increase in pressure caused by high-frequency vibration [2]. Due to the role of internal heat, different natural frequency parts are excited, it will cause vibration, which radiate a strong combustion noise.

The combustion rate of marine diesel engines has a significant impact on the power and economy of diesel engines. Diesel engine combustion process can usually be divided into the following stages: fire delay stage, rapid combustion stage, slow combustion stage and the latter 
stage of combustion. The first stage of the maintenance time is shorter, usually in the $0.0003-0.0007 \mathrm{~s}$, in the process to provide important conditions for the latter part of the chemical reaction; in the second stage, the fuel within the cylinder began to burn quickly, the cylinder wall and In the slow combustion stage, the piston has begun to move down, the fuel in the cylinder has been burned out, the temperature and pressure inside the cylinder are also in the same direction, In the rapid decline, this time will not form a clear vibration. By further study of the combustion noise mechanism, it is found that the rapid combustion stage and the slow combustion stage are the main stages of noise generation, and this noise is due to the high frequency of heat flow and pressure driven. So the control of the combustion noise is actually an effective control of the average pressure rise rate [3].

\section{Control and Emission of Vibration and Noise of Marine Diesel Engine}

The main sources of vibration and noise of marine diesel engines are noise, noise generated by combustion noise and airflow. The reasons for the combustion noise are more complicated. In order to further improve the stability of the working of the diesel engine, the following precaution measures are put forward for the combustion noise. The selection and design of the combustion chamber are strengthened and the fuel supply advance angle is reduced. The double spring injector and pressurization technology are used to improve the fuel pump pressure.

Selection and Design of Combustion Chamber. The structure of the combustion chamber of the marine diesel engine has an important effect on the operation of the whole equipment. In fact, the diesel engine combustion chamber used has direct and split type. Among them, the direct injection of combustion chamber can be divided into open, semi-open and spherical combustion chamber, separated mainly eddy current and pre-combustion type two. Through the actual use found that spherical, eddy current and pre-combustion structure is better, resulting in lower noise. Therefore, in actual use, should be based on the project cost and the actual situation of the analysis, so as to choose the appropriate combustion chamber structure, improve the stability of diesel engine work [4]. In the study, through comparison found that the combustion chamber structure and noise there is a certain connection between the larger diameter of the combustion chamber, the lower the depth, the higher the possibility of noise generated. On the contrary, the noise is small and the emissions are better. Therefore, in the design of marine diesel engine combustion chamber, the cost conditions permit, should be based on the results of the study as far as possible the choice of less noise combustion chamber structure.

Reduce Fuel Supply Advance Angle. The control of the fuel supply has an important effect on the combustion situation in the combustion chamber. The oil feed advance angle is an important parameter to control the oil intake. It can reduce the emission of nitrogen oxides and reduce the noise by reasonable adjustment. It is found in the experiment that the oil feed advance angle can be reduced to achieve the above purpose, but this parameter should be based on equipment's normal work.

Use a Double Spring Injector. By using the dual spring injector to control the nozzle, you can effectively reduce the occurrence of combustion noise. In the actual marine diesel engine equipment, the use of dual-spring injector, at the beginning of work, the fuel pump in a high-pressure environment, the injector to change the traditional one jet mode, into two eject mode, and the first part only a small amount of the fuel, the second step will be the remaining large amount of fuel for discharge [5]. The use of this control method can make the injector pressure rise rate become more gentle so as to achieve the purpose of reducing noise.

Use Pressurization Technology. The use of pressurization in marine diesel equipment can improve the ignition conditions of the mixture in the cylinder, thereby shortening the ignition delay phase. Experiments show that the use of pressurization technology can significantly reduce the noise.

Increase the Pump Side Pressure of the Fuel Injection Pump. The combustion efficiency of the combustion chamber of a marine diesel engine has a direct effect on the generation of noise. When the pressure of the fuel injection pump is small, the atomizer of the injector is low, resulting 
in uneven mixing with the air, which cannot provide favorable conditions for the combustion of the combustion chamber, causing local deflagration and causing noise vibration. Therefore, by increasing the pump-side pressure of the fuel injection pump can effectively improve the atomization situation, thereby achieving the purpose of reducing noise [6].

\section{Case Analysis of Vibration and Noise of Marine Diesel Engine}

In order to understand the vibration and noise in a more in-depth way, this part analyzes the form and cause of the vibration and noise failure in detail with the concrete case, and gives the concrete measures to eliminate the trouble.

Failure Performance. A ship diesel engine in normal operation has the following two failures.

Failure 1: Tire mainframe in normal operation, the speed in a certain range of control, when the speed exceeds 300r / min above, after a certain period of time, the engine oil temperature exceeds $35{ }^{\circ} \mathrm{C}$, the device began to show an irregular, noisy percussion, As well as the burning of the percussion. At this time the speed is affected by the vibration, there is about 10r / min fluctuations, and the frequency of change quickly; At this time of the exhaust temperature, although within the effective range, but relative to the normal circumstances, the temperature increase, The sound of the operation of the subtle changes in the sound. The main source of the noise is the valve mechanism, the frequency due to the different speed show a change in the form; Failure 2: marine diesel engine in non-specific circumstances, the random emergence of high-speed rack movement, while diesel engine vibration. The noise is very large, and very complex, the speed also appears from top to bottom $10 \sim 20 \mathrm{r} / \mathrm{min}$ fluctuations [7].

Troubleshooting. Exclusion of Failure 1. Through the ear hearing method and further research, it is found that the noise in fault 1 may originate from the valve structure. The main reasons for this phenomenon are as follows: First, the gap of the valve is not strictly designed according to the relevant requirements; Second, the valve internal equipment mechanical damage; Third, the cam loosening or abnormal wear [8]. The above structure is further checked on the basis of the above possibilities. First check the camshaft, the main check whether there is tolerance of bearing clearance, partial grinding and other issues, the final conclusion is that there is no exception; after the valve system to check the external components, tappet and guide between the tube The gap did not find obvious anomalies, the parts after the clean-up after re-assembly, after testing found that noise has weakened, but the noise situation is not eliminated; to further lock the cause of the failure of the diesel fuel system to check the cause of the second failure due to the impact of high-pressure pump, resulting in uneven oil supply between the various cylinders; the same time, the fuel injection is not the same as the following: First, the fuel injection timing is not allowed to lead to the order of the fire there is a certain difference; Third, because the fuel injector work did not meet the standard, resulting in the combustion between the various cylinders there is a big difference; Finally, check the diesel engine governor, and the governor's work is mainly reflected in the fuel supply Irregular changes, resulting in abnormal combustion of the cylinder and then produce abnormal noise. Then, check the accuracy and sensitivity of the governor, and no abnormality is found [9].

Through the above screening analysis, determine the main source of failure is the valve structure, although there are some other problems, but not enough to have a greater impact on the overall noise. And these gas distribution structure in the actual work has lost a certain regularity. Through further study found that the source of the fault is the camshaft transmission gear and the shaft between the interference fit slight loosening, shaft and transmission gear between the structure shown in Fig 1. 


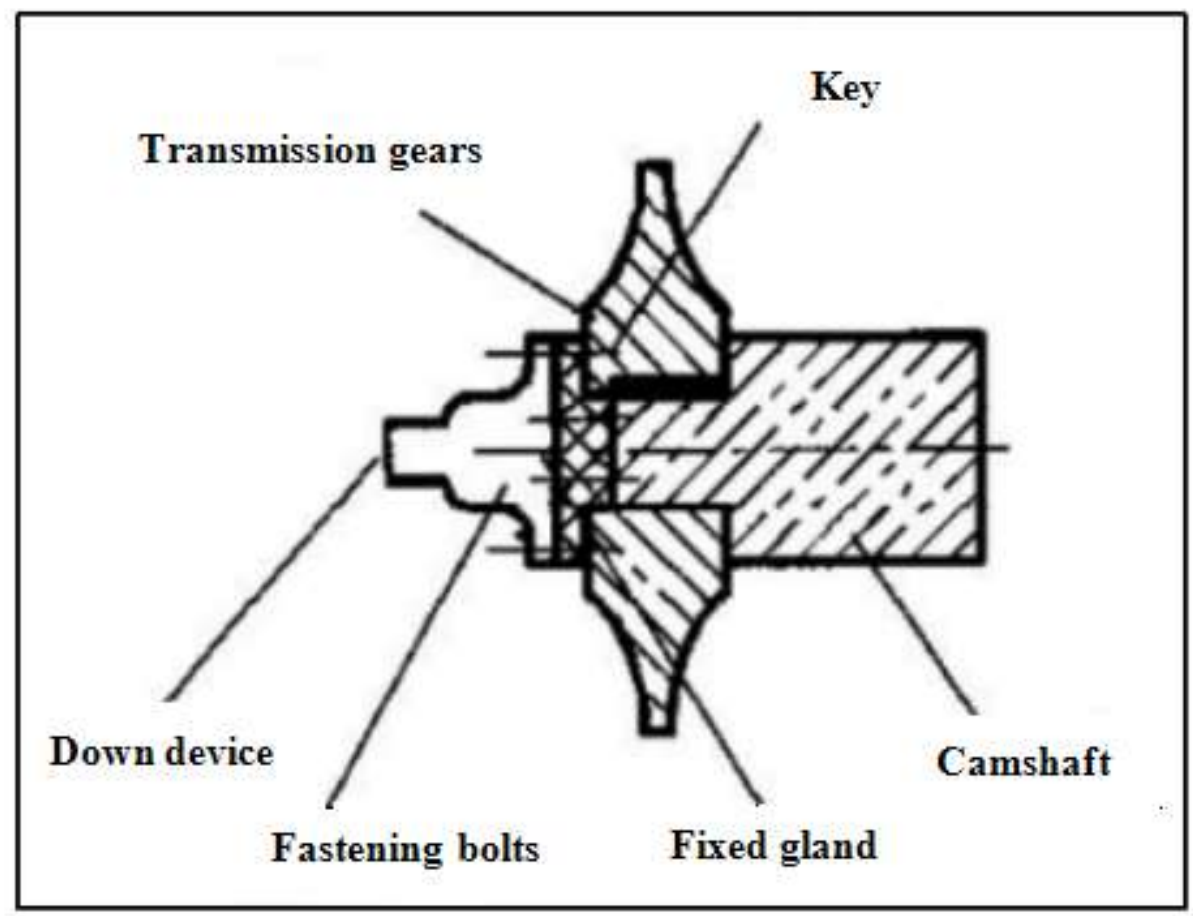

Fig. 1 Matching of Cam Shaft and Drive Gear

In order to further understand the source of noise failure, the demolition of diesel equipment. After dismantling, it was found that the gap between the camshaft and the transmission gear was slightly loosened, and irregularities were also observed on the side of the panel. In the cold conditions, the speed of 300r / min below, you can ensure the normal work. But in the hot state when the speed exceeds 300r/ min, due to thermal expansion and contraction caused by the failure, the structure of the operation of the law, the diesel engine work disorders, thus showing a more obvious noise [10]. In the specific course of navigation, the noise of the ship's diesel engine will also change with the load changes. Finally, through the damage and easy aging components of the replacement so that the internal structure returns back to become normal.

Analysis of the Cause of Failure 2.Through the observation of the structure of the marine diesel engine, it was found that the diesel engine was connected in series with a generator and a water supply pump. The failure is also in the high-pressure pump replacement, the equipment running after a period of time. Through the analysis and research, the main reason for the noise failure is the base replacement after the fixed bolt and pump plate there is no match between the situation. This does not match in a certain load range, the performance of the fault is small, not easy to be aware of. With the increase in load and the use of time to deepen, this situation continues to intensify, and then lead to greater noise. In the process of fluctuation, the high-pressure pump camshaft and the drive shaft of the different heart problems, resulting in governor vibration enhancement, noise becomes more obvious.

Do a Good Job in Failure Risk Analysis. Identify risk targets and scope, develop effective risk identification programs, collect data collectively, field survey analysis, and submit identification results. Select the appropriate risk identification methods, such as brainstorming, fault tree and checklist method. Through the risk identification for managers to provide a comprehensive risk factor awareness, divided according to different stages, to identify the risk factors at each stage. The risk investigation process attaches great importance to the experience provided by the professional, effectively discusses the project part of the staff feedback analysis of experts, and finally complete the data integration and classification for the latter part of the risk management and response to lay the foundation. And there is a need for risk-prone phenomenon of the implementation of the focus of the workplace to address the risk database information, the location of the risk point, type, the reasons should be accurately recorded, while determining a number of different risk points specific Location, type and cause of the implementation of the risk of pre-control, and then to achieve the 
risk of investigation and assessment of the operation. In addition to ensure that the oil pipeline cleaning is essential for the oil pipeline appears in the garbage and impurities to be cleaned in time to ensure that the engine body and bearing clean. Therefore, after the repair, the unit by the system can be used to clean the way the oil cycle.

\section{Conclusion}

In summary, the noise in marine diesel engines is an important pollution in industrial development. In the environment of saving the environment of environment-friendly economy, it is very important to improve the stability of diesel engine equipment by adopting certain measures to improve the quality of equipment and enhance the control technology. In this study, the specific cases, the analysis of ship diesel engine vibration and noise failure measures. It is hoped that this article will provide the experience and reference for front-line workers.

\section{References}

[1] Gao Hande, Analysis on the Misunderstanding of Marine Diesel Engine 's Daily Management [J]. Heilongjiang Science and Technology Information.2014(30):123-124.

[2] Zuo Peizhu. Analysis on the Current Situation of Marine Diesel Engine Management and Maintenance [J]. Guide to Get Rich with Science and Technology. 2014(12):98.

[3] Yang Zilong. Development Trend of Energy Saving and Emission Reduction Technology for Marine Diesel Engine [J]. Diesel Engine.2016(03):67-68.

[4] Shi Zhigang, Fan Jianxin. Development of Diesel Engine in Inland River [J]. Diesel Engine.2015(01):101-102.

[5] Wang Yonghong. Cause Analysis and Preventive Measures of Pore Defects in Camshaft Shaft Holes of Certain Marine Diesel Engine [J]. Thermal Processing Technology.2017(13):74.

[6] Pan Yuping, Wan Longjun. Virtual Disassembly Simulation of Marine Diesel Engine [J]. Traffic Information and Security.2014(02):23.

[7] Chang Tie, Yang Puze. Study on Tribological Properties of Cylinder Liner Based on Synergistic Effect of Surface Thermal Coating and Surface Texture [J]. Thermal Processing Technology.2017(14):95.

[8] Liu Haifeng, Zhang Huixiang, Wang Hu, Zou Xian. Effect of Air Intake Component on NOx Emission from Low Speed Ship [J]. Combustion Science and Technology.2017(04):81.

[9] Zhu Jun. Study on Application of Thermal Power Monitoring Technology for Main Bearing Wear of Marine Diesel Engine [J]. Diesel Engine.2017(03):98.

[10] Ma Tianshuai. Optimization of Diesel Engine Intercooler Flow Uniformity Based on CFD Simulation [J]. Diesel Engine.2017(03):72. 